\title{
Corrigendum: A Practical Guide to Resonance Frequency Assessment for Heart Rate Variability Biofeedback
}

\author{
Fred Shaffer ${ }^{1 *}$ and Zachary M. Meehan ${ }^{2}$ \\ ${ }^{1}$ Center for Applied Psychophysiology, Truman State University, Kirksville, MO, United States, ${ }^{2}$ Department of Psychological \\ and Brain Sciences, University of Delaware, Newark, DE, United States
}

Keywords: biofeedback, complexity, emotional self-regulation, heart rate variability, neurocardiology, resonance, performance

\section{A Corrigendum on}

A Practical Guide to Resonance Frequency Assessment for Heart Rate Variability Biofeedback by Shaffer, F., and Meehan, Z. M. (2020). Front. Neurosci. 14:570400. doi: 10.3389/fnins.2020.570400

In the original article, there was an error. The high-frequency band's upper limit was reversed.

A correction has been made to "A Resonance Frequency Assessment Protocol section," "Terms and Definitions subsection."

Data from heart rate, respiration, and their synchrony provide detailed information for resonance frequency assessment. Clinicians who assess resonance frequency are concerned with the smoothness and regularity of heart rate signals. Heart rate-respiration phase synchrony is the phase angle of the peaks and troughs of the heart rate and respiration rate signals. HRV frequency-domain metrics calculate the spectral distribution of signal energy. HRV biofeedback is concerned with the very-low-frequency (VLF; $0.0033-0.04 \mathrm{~Hz}$ ), low-frequency (LF; $0.04-0.15 \mathrm{~Hz}$ ), and high-frequency

OPEN ACCESS

Approved by:

Frontiers Editorial Office,

Frontiers Media SA, Switzerland

*Correspondence:

Fred Shaffer

fredricshaffer@gmail.com

Specialty section:

This article was submitted to

Autonomic Neuroscience,

a section of the journal

Frontiers in Neuroscience

Received: 09 November 2020 Accepted: 13 November 2020 Published: 01 December 2020

Citation:

Shaffer F and Meehan ZM (2020) Corrigendum: A Practical Guide to

Resonance Frequency Assessment for Heart Rate Variability Biofeedback.

Front. Neurosci. 14:627512.

doi: $10.3389 /$ fnins.2020.627512 $(\mathrm{HF} ; 0.15-0.40 \mathrm{~Hz})$ bands. Although there is uncertainty regarding the sources of VLF power in short-term measurements (Kleiger et al., 2005), sympathetic activation due to effortful breathing is a possible source (Bernardi et al., 1996). There is also disagreement about the sources of LF power in short-term measurements (Akselrod et al., 1981; Goldstein et al., 2011; Reyes del Paso et al., 2013). LF power is an important indicator of HRV biofeedback training success for several reasons. First, slow paced breathing, which is one method of stimulating the baroreflex, increases LF power by increasing cardiac vagal tone (Kromenacker et al., 2018). Second, increased LF power is associated with greater RSA and HRV (Vaschillo et al., 2002). Increased RSA and HRV occur in the LF range because the baroreceptor reflex's resonance frequency resides within this range (Lehrer et al., 2020). HF power is due to parasympathetic activity, and the natural logarithm of HF power indexes cardiac vagal tone (Task Force Report, 1996). Absolute power is the signal energy within a frequency band expressed in $\mathrm{ms}^{2} / \mathrm{Hz}$. Normalized power is the percentage of total power. For example, normalized LF power is $\mathrm{LF} /(\mathrm{LF}+\mathrm{HF})$ or LF/(VLF + LF + HF). Peaks are the highest-amplitude frequencies within a band like LF. Resonance frequency assessment examines both the magnitude and number of LF peaks (Shaffer and Ginsberg, 2017). LF peak amplitude and the number of LF peaks are among six resonance frequency selection criteria (Lehrer et al., 2013). Larger peaks indicate greater resonance effects due to increased breathing and heart rate synchrony. Several LF peaks may occur when individuals breathe at a single rate outside of their resonance frequency. This can produce separate peaks at the baroreflex frequency and their actual respiratory rate. While breathing at adjacent rates may still stimulate the baroreflex, it will produce smaller resonance effects (Lehrer et al., 2020). When clients do not precisely follow instructions, changing respiration rates can also produce multiple peaks and weaker resonance effects. Breathing 
in a narrow frequency range around the resonance frequency better stimulates the baroreflex and increases RSA than breathing in a wider frequency range (Vaschillo et al., 2002).

\section{REFERENCES}

Akselrod, S., Gordon, D., Ubel, F. A., Shannon, D. C., Barger, A. C., and Cohen, R. J. (1981). Power spectrum analysis of heart rate fluctuation: aquantitative probe of beat-to-beat cardiovascular control. Science 213, 220-222. doi: 10.1126/science.6166045

Bernardi, L., Valle, F., Coco, M., Calciati, A., and Sleight, P. (1996). Physical activity influences heart rate variability and very-low-frequency components in Holter electrocardiograms. Cardiovasc. Res. 32, 234-237. doi: 10.1016/0008-6363(96)00081-8

Goldstein, D. S., Bentho, O., Park, M. Y., and Sharabi, Y. (2011). Low frequency power of heart rate variability is not a measure of cardiac sympathetic tone but may be a measure of modulation of cardiac autonomic outflows by baroreflexes. Exp. Physiol. 96, 1255-1261. doi: 10.1113/expphysiol.2010.056259

Kleiger, R. E., Stein, P. K., and Bigger, J. T. Jr. (2005). Heart rate variability: measurement and clinical utility. Ann. Noninvasive Electrocardiol. 10, 88-101. doi: 10.1111/j.1542-474X.2005.10101.x

Kromenacker, B. W., Sanova, A. A., Marcus, F. I., Allen, J. J. B., and Lane, R. D. (2018). Vagal mediation of low-frequency heart rate variability during slow yogic breathing. Psychosom. Med. 80, 581-587. doi: $10.1097 /$ psy. 0000000000000603

Lehrer, P., Vaschillo, B., Zucker, T., Graves, J., Katsamanis, M., Aviles, M., et al. (2013). Protocol for heart rate variability biofeedback training. Biofeedback 41, 98-109. doi: 10.5298/1081-5937-41.3.08
The authors apologize for this error and state that this does not change the scientific conclusions of the article in any way. The original article has been updated.

Lehrer, P. M., Vaschillo, E., and Vidali, V. (2020). Heart rate and breathing are not always in phase during resonance frequency breathing. Appl. Psychophysiol. Biofeedback 45, 145-152. doi: 10.1007/s10484-020-09459-y

Reyes del Paso, G. A., Langewitz, W., Mulder, L. J. M., Van Roon, A., and Duschek, S. (2013). The utility of low frequency heart rate variability as an index of sympathetic cardiac tone: a review with emphasis on a reanalysis of previous studies. Psychophysiology 50, 477-487. doi: 10.1111/psyp.12027

Shaffer, F., and Ginsberg, J. P. (2017). An overview of heart rate variability (HRV) metrics and norms. Front. Public Health 5:258. doi: 10.3389/fpubh.2017.00258

Task Force Report (1996). Heart rate variability: standards of measurement, physiological interpretation, and clinical use. Circulation 93, 1043-1065. doi: 10.1161/01.CIR.93.5.1043

Vaschillo, E., Lehrer, P., Rishe, N., and Konstantinov, M. (2002). Heart rate variability biofeedback as a method for assessing baroreflex function: a preliminary study of resonance in the cardiovascular system. Appl. Psychophysiol. Biofeedback 27, 1-27. doi: 10.1023/A:10145873 04314

Copyright (c) 2020 Shaffer and Meehan. This is an open-access article distributed under the terms of the Creative Commons Attribution License (CC BY). The use, distribution or reproduction in other forums is permitted, provided the original author(s) and the copyright owner(s) are credited and that the original publication in this journal is cited, in accordance with accepted academic practice. No use, distribution or reproduction is permitted which does not comply with these terms. 